K. Ueno

Nagoya Math. J.

Vol. 99 (1985), 111-130

\title{
PROJECTIVE STRUCTURES OF ELLIPTIC DIFFERENTIAL OPERATORS
}

\author{
KAZUSHIGE UENO \\ Dedicated to Professor Nobuo Shimada on his 60-th birthday
}

\section{Introduction}

In the study of differential equations from the standpoint of the automorphism pseudogroups, the differential invariants of the pseudogroups play an important role.

A general study of pseudogroups and their differential invariants originated with Sophus Lie. He applied his study to the classification of ordinary and partial differential equations. So as to study differential equations from his point of view, it is very important to write the given differential equation by the differential invariants of the automorphism pseudogroup. That is to say, the geometric structure of a differential equation is contained in the expression of the equation by its differential invariants.

In this paper we shall deal with elliptic systems of differential equations which admit the automorphism pseudogroup generated by the maximal subgroup $G$ of the affine transformation group of the $m$-dimensional linear space $R^{m}$ whose linear part is the center of $G L(m, R)$.

These systems of differential equations will be proved to admit projective structures of some type. Thus elliptic systems of differential equations whose automorphism pseudogroups are generated by $G$ will be called projective.

In Section 1 we study the automorphism pseudogroups of elliptic differential equations of second order. There we shall show that they are generated by subgroups of the affine transformation group of $R^{m}$ (Proposition 3.1).

In Section 2 we investigate canonical generators of projective elliptic

Received June 4, 1984. 
differential equations (Theorem 4.5). These generators are families of differential invariants of the automorphism psuedogroups and involve the geometric structures of the differential equations.

In Section 3 we deal with equivalence problem of projective elliptic differential equations (Theorem 5.2, Corollary 6.2). We shall see that these equivalence problems are reduced to those of a kind of projective geometry (Remark 5.1).

In Section 4 we state propositions which present examples of projective elliptic differential operators of second order (Proposition 8.1, Proposition 8.2). In the calculation of the automorphism pseudogroups of these differential operators, the pseudoinvolutiveness (Definition 2.1) will play an important role.

\section{$\S 1$. Automorphism pseudogroups of elliptic differential operators}

1. Let $N$ be the set of non-negative integers and denote by $p=$ $\left(p_{1}, \cdots, p_{n}\right) \in N^{n}(n \geq 2)$ a multi-index. For the canonical coordinate system $\left\{x_{1}, \cdots, x_{n}\right\}$ on $R^{n}$, we set

$$
D^{p}=\left(\frac{\partial}{\partial x_{1}}\right)^{p_{1}}\left(\frac{\partial}{\partial x_{2}}\right)^{p_{2}} \cdots\left(\frac{\partial}{\partial x_{n}}\right)^{p_{n}}
$$

and denote by $|p|=p_{1}+\cdots+p_{n}$ the order of $D^{p}$. A differential operator is an expression

$$
P(x, D)=\sum_{|p| \leq m} a_{p}(x) D^{p},
$$

where the coefficients $a_{p}(x)$ are $R$-valued $C^{\infty}$-functions on $R^{n}$. To this operator, for a fixed $x$, there corresponds a polynomial $P(x, \xi)=\sum_{|p| \leq m} a_{p}(x) \xi^{p}$ where $\xi=\left(\xi_{1}, \cdots, \xi_{n}\right) \in R^{n}$ and $\xi^{p}=\xi_{1}^{p_{1}} \xi_{2}^{p_{2}} \cdots \xi_{n}^{p_{n}}$. The principal part of $P(x, D)$ is defined by

$$
P_{\langle m\rangle}(x, D)=\sum_{|p|=m} a^{p}(x) D^{p} .
$$

and $P_{\langle m\rangle}(x, \xi)=\sum_{|p|=m} a(x) \xi^{p}$ is called the symbol of $P(x, D)$.

Let $E$ be a vector space over $R$ and denote by $\Gamma\left(R^{n}, E\right)$ the set of local $C^{\infty}$-maps of $R^{n}$ to $E$. Let $F$ be another vector space over $R$ and denote by $\operatorname{Hom}(E, F)$ the set of linear maps. Then a differential operator of $\Gamma\left(R^{n}, E\right)$ to $\Gamma\left(R^{n}, F\right)$ is an expression

$$
P(x, D)=\sum_{|p| \leq m} a_{p}(x) D^{p}
$$


where the $a_{p}(x)$ are $\operatorname{Hom}(E, F)$-valued $C^{\infty}$-functions on $R^{n}$. To this differential operators, there corresponds the principal part $P_{\langle m\rangle}(x, D)=$ $\sum_{|p|=m} a_{p}(x) D^{p}$ of $P(x, D)$ or the symbol $P_{\langle m\rangle}(x, \xi)=\sum_{|p|=m} a_{p}(x) \xi^{p}$ of $P(x, D)$.

Definition 1.1. A differential operator $P(x, D)=\sum_{|p| \leq m} a_{p}(x) D^{p}$ of $\Gamma\left(R^{n}, E\right)$ to $\Gamma\left(R^{n}, F\right)$ is called elliptic if $\operatorname{dim} E=\operatorname{dim} F$ and $P_{\langle m\rangle}(x, \xi)=$ $\sum_{|p|=m} a_{p}(x) \xi^{p}$ is an invertible element of $\operatorname{Hom}(E, F)$ for any $x \in R^{n}$ and any non-zero vector $\xi \in R^{n}$.

2. Let $P(x, D)=\sum_{|p| \leq m} a_{p}(x) D^{p}$ be a differential operator of $\Gamma\left(R^{n}, E\right)$ to $\Gamma\left(R^{n}, F\right)$. Denote by $J^{k}\left(R^{n}, E\right)$ the space of $k$-jets of local maps of $R^{n}$ to $E$. Then it is easy to see that there exists a unique map $\sigma: J^{k}\left(R^{n}, E\right)$ $\rightarrow \Gamma\left(R^{n}, F\right)$ such that $P(x, D) \varphi=\sigma\left(j_{x}^{k}(\varphi)\right)$ for any element $\varphi \in \Gamma\left(R^{n}, E\right)$.

Denote by $0 \in \Gamma\left(R^{n}, F\right)$ the zero map of $R^{n}$ to $F$ and set $I(P)=\sigma^{-1}(0)$ $\subset J^{k}\left(R^{n}, E\right)$. Let $\mathscr{S}(P) \subset \Gamma\left(R^{n}, E\right)$ denote the set of local solutions of $P(x, D)$ and set $S(P)=\left\{j_{x}^{k}(\varphi) ; x \in\right.$ the domain of $\left.\varphi, \varphi \in \mathscr{S}(P)\right\} \subset J^{k}\left(R^{n}, E\right)$. Then, in general, we have $S(P) \subset I(P)$.

Definition 2.1. A differential operator $P(x, D)=\sum_{|p| \leq m} a_{p}(x) D^{p}$ is called pseudoinvolutive if $S(P)=I(P)$.

If $P(x, D)$ is elliptic, then the system of differential equations

$$
\sum_{|p| \leq m} a_{p}(x) D^{p} \varphi=0
$$

can be rewritten by a system of Cauchy-Kowalewski type Therefore if $P(x, D)$ is elliptic and analytic, we get $I(P)=S(P)$.

For an open subset $\mathcal{O}$ of $E$, we set $\mathscr{S}(P) \mid \mathcal{O}=\{s \in \mathscr{S}(P) ; \operatorname{Im} s \subset \mathcal{O}\}$.

Definition 2.2. Two differential operators $P_{1}(x, D)$ and $P_{2}(x, D)$ are said to be locally isomorphic at $\left(z_{1}, z_{2}\right) \in E \times E$ if there exists a $C^{\infty}$ diffeomorphism $\phi$ of a neighbourhood $\mathscr{U}$ of $z_{1}$ to a neighbourhood $\mathscr{V}$ of $z_{2}$ such that $\phi\left(\mathscr{S}\left(P_{1}\right) \mid \mathscr{U}\right)=\mathscr{S}\left(P_{2}\right) \mid \mathscr{V}$. $\phi$ is called a local isomorphism of $P_{1}(x, D)$ to $P_{2}(x, D)$ at $\left(z_{1}, z_{2}\right)$. If $P_{1}(x, D)=P_{2}(x, D)=P(x, D)$ and $z_{1}=z_{2}=z$, then a local isomorphism of $P(x, D)$ to $P(x, D)$ at $(z, z)$ is called a local automorphism of $P(x, D)$ at $z$. Denote by $\mathscr{A}_{z}(P)$ the set of local automorphisms of $P(x, D)$ at $z$. If there is no confusion, $\mathscr{A}_{z}(P)$ is denoted simply by $\mathscr{A}(P)$. $\mathscr{A}(P)$ is called the automorphism pseudogroup of $P(x, D)$.

3. Assume that a differential operator $P(x, D)=\sum_{|p| \leq 2} a^{p}(x) D^{p}$ of $\Gamma\left(R^{n}, E\right)$ to $\Gamma\left(R^{n}, F\right)$ is elliptic and pseudoinvolutive. Choose a coordinate system $\left\{u_{1}, \cdots, u_{m}\right\}$ (resp. $\left.\left\{v_{1}, \cdots, v_{m}\right\}\right)$ on $E$ (resp. $F$ ). Then for $u \in \Gamma\left(R^{n}, E\right)$ 
and $v \in \Gamma\left(R^{n}, F\right)$, the relation $v=P(x, D) u$ can be written by

$$
\begin{gathered}
v_{i}=\sum_{j=1}^{m} \sum_{k, \ell=1}^{n} a_{k \ell}^{i j}(x) \frac{\partial^{2} u_{j}}{\partial x_{k} \partial x_{\ell}}+\sum_{j=1}^{m} \sum_{k=1}^{n} b_{k}^{i j}(x) \frac{\partial u_{j}}{\partial x_{k}}+\sum_{j=1}^{m} c^{i j}(x) u_{j} . \\
(i=1, \cdots, m) .
\end{gathered}
$$

If $u: R^{n} \supset \mathscr{U} \rightarrow E$ belongs to $\mathscr{S}(P)$ and $\phi: u(\mathscr{U}) \rightarrow E$ belongs to $\mathscr{A}(P)$, then

$$
\begin{gathered}
\sum_{j=1}^{m} \sum_{k, \ell=1}^{n} a_{k \ell}^{i j}(x) \frac{\partial^{2} u_{j}}{\partial x_{k} \partial x_{\ell}}+\sum_{j=1}^{m} \sum_{k=1}^{n} b_{k}^{i j}(x) \frac{\partial u_{j}}{\partial x_{k}}+\sum_{j=1}^{m} c^{i j}(x) u_{j}=0 \\
(i=1, \cdots, m)
\end{gathered}
$$

and

$$
\begin{gathered}
\sum_{j=1}^{m} \sum_{k, \ell=1}^{n} a_{k \ell}^{i j}(x)\left(\sum_{\alpha=1}^{m} \frac{\partial \phi_{j}}{\partial u_{\alpha}}(u) \frac{\partial^{2} u_{\alpha}}{\partial x_{k} \partial x_{\ell}}+\sum_{\beta, \gamma=1}^{m} \frac{\partial^{2} \phi_{j}}{\partial u_{\beta} \partial u_{\gamma}}(u) \frac{\partial u_{\beta}}{\partial x_{k}} \frac{\partial u_{r}}{\partial x_{\ell}}\right) \\
+\sum_{j=1}^{m} \sum_{k=1}^{n} b_{k}^{i j}(x)\left(\sum_{\delta=1}^{m} \frac{\partial \phi_{j}}{\partial u_{\delta}}(u) \frac{\partial u_{\delta}}{\partial x_{k}}\right)+\sum_{j=1}^{m} c^{i j}(x) \phi_{j}(u)=0 \\
(i=1, \cdots, m) .
\end{gathered}
$$

Since $P(x, D)$ is elliptic, $\operatorname{det}\left(a_{k \ell}^{i j}(x)\right)_{1 \leq i, j \leq m} \neq 0$. Therefore from (3.1) and (3.2), we get as the system of defining equations of $\mathscr{A}(P)$

$$
\begin{gathered}
\sum_{j=1}^{m} \sum_{k, \ell=1}^{n} a_{k l}^{i j}(x)\left(\sum_{\beta, r=1}^{m} \frac{\partial^{2} \phi_{j}}{\partial u_{\beta} \partial u_{r}}(u) \frac{\partial u_{\beta}}{\partial x_{k}} \frac{\partial u_{r}}{\partial x_{\ell}}\right) \\
+\mathscr{K}^{i}\left(x, u, \cdots, \frac{\partial u_{\hat{o}}}{\partial x_{h}}, \cdots, \frac{\partial^{2} u_{\alpha}}{\partial x_{k} \partial x_{\ell}}, \cdots\right)=0 \\
(i=1, \cdots, m)
\end{gathered}
$$

on $S(P)=I(P)$ where $\mathscr{K}^{i}$ is linear with respect to $\partial u_{\delta} / \partial x_{h}(\delta=1, \cdots, m$; $h=1, \cdots, n)$ and $\partial^{2} u_{\alpha} / \partial x_{k} \partial x_{\ell}(\alpha=1, \cdots, m ; k, \ell=1, \cdots, n)$ and independent of $\partial^{2} u_{\alpha} / \partial x_{1}^{2}(\alpha=1, \cdots, m)$. Furthermore $\partial u_{\delta} / \partial x_{h}(\delta=1, \cdots, m ; h=1$, $\cdots, n)$ and $\partial^{2} u_{\alpha} / \partial x_{k} \partial x_{\ell}(\alpha=1, \cdots, m ; k, \ell=1, \cdots, n$ and $k$ or $\ell \neq 1)$ are independent on $I(P)=S(P)$. Therefore we get $\sum_{j=1}^{m} a_{k \ell}^{i j}(x)\left(\partial^{2} \phi_{j} / \partial u_{\beta} \partial u_{r}\right)=0$ $(i=1, \cdots, m)$. The ellipticity of $P(x, D)$ means that $\partial^{2} \phi_{j} / \partial u_{\beta} \partial u_{r}=0(j, \beta$, $\gamma=1, \cdots, m)$. This has proved

Proposition 3.1. For any elliptic pseudoinvolutive differential operator $P(x, D)=\sum_{|p| \leq 2} a_{p}(x) D^{p}, \mathscr{A}(P)$ is generated by a subgroup of the affine transformation group on $E$.

We denote by $\mathscr{A}_{0}(P)$ the set of elements of $\mathscr{A}(P)$ which are defined globally on $E$. Then $\mathscr{A}_{0}(P)$ is a group and $\mathscr{A}(P)$ is generated by $\mathscr{A}_{0}(P)$. 
Definition 3.1. Let $P(x, D)$ be any differential operator of $\Gamma\left(R^{n}, E\right)$ to $\Gamma\left(R^{n}, F\right)$. If the automorphism pseudogroup $\mathscr{A}_{2}(P)$ is generated by the maximal subgroup $G$ of the affine transformation group on $E$ whose linear part is the center of $G L(E), P(x, D)$ is said to be projective at $z$. Since $G$ is transitive on $E$, it is clear that, if $P(x, D)$ is projective at a point $z$, then it is projective at any point of $E$. Therefore $P(x, D)$ is then simply said to be projective.

Note that, from (3.1) and (3.2), the projectivity of $P(x, D)$ implies $c^{i j}(x) \equiv 0(i, j=1, \cdots, m)$.

\section{§ 2. Canonical generators of projective elliptic differential operators}

4. Let $N$ and $Q$ be any two $C^{\infty}$-manifolds and denote by $J^{k}(N, Q)$ the space of $k$-jets of local maps of $N$ to $Q$. Let $\Gamma$ be a pseudogroup on $Q$ and let $\phi \in \Gamma$. Define a local transformation $\phi^{(k)}$ on $J^{k}(N, Q)$ by $\phi^{(k)}\left(j_{x}^{k}(f)\right)$ $=j_{x}^{k}(\phi \circ f)$ and denote by $\Gamma^{(k)}$ the pseudogroup on $J^{k}(N, Q)$ generated by $\left\{\phi^{(k)} ; \phi \in \Gamma\right\} . \quad \Gamma^{(k)}$ is called the $k$-th prolongation of $\Gamma$ to $J^{k}(N, Q)$.

Let $\mathscr{L}$ be a weak Lie algebra sheaf on $Q$ ([1]). Then, by the prolongation of the pseudogroup $\mathscr{P}(\mathscr{L})$ generated by $\mathscr{L}, \mathscr{L}$ is prolonged to a sheaf of vector fields $\mathscr{L}^{(k)}$ on $J^{k}(N, Q)$. Let $\mathscr{L}_{p}^{(k), 0}$ denote the isotropy of the stalk $\mathscr{L}_{p}^{(k)}$ and set $D_{p}^{(k)}=\mathscr{L}_{p}^{(k)} / \mathscr{L}_{p}^{(k), 0}$.

Definition 4.1. $\mathscr{L}$ is said to be $N$-regular at $(x, f)$ if, for any integer $k$, the correspondence $D^{(k)}: J^{k}(N, Q) \ni p \rightarrow D_{p}^{(k)} \subset T_{p}\left(J^{k}(N, Q)\right)$ defines an involutive distribution on a neighbourhood of $j_{x}^{k}(f)$.

Denote by $E$ the vector space $R^{m}$ and let $\left\{z_{1}, \cdots, z_{m}\right\}$ be the canonical coordinate system on $E$. Also denote by $\left\{x_{1}, \cdots, x_{n}\right\}$ the canonical coordinate system on $R^{n}$. Then we have the coordinate system

$$
\left\{x_{1}, \cdots, x_{n}, z_{1}, \cdots, z_{m}, \cdots, p_{j}^{i}, \cdots, p_{j_{1} j_{2}}^{i}, \cdots\right\}
$$

on $j^{k}\left(R^{n}, E\right)$ such that

$$
p_{j_{1} \cdots j_{\alpha}}^{2}\left(j_{x}^{\alpha}(f)\right)=\frac{\partial^{\alpha} z_{j} \circ f}{\partial x_{j_{1}} \cdots \partial x_{j_{\alpha}}}\left(1 \leq i \leq m ; 1 \leq \alpha \leq k ; 1 \leq j_{h} \leq n\right) .
$$

For any vector field $X$ on $E$, denote by $X^{(k)}$ the $k$-th prolongation of $X$ to $J^{k}\left(R^{n}, E\right)$.

Lemma 4.1. Let $X$ be such a vector field on $E$ that $X=\sum_{i=1}^{m}\left(a z_{i}+b_{i}\right)$ $\times \partial / \partial z_{i}$ where $a$ and $b_{i} \in R$. Then 


$$
\begin{aligned}
X^{(k)}= & a\left(\sum_{i=1}^{m} z_{i} \frac{\partial}{\partial \boldsymbol{z}_{i}}+\sum_{i=1}^{m} \sum_{j=1}^{n} p_{j}^{i} \frac{\partial}{\partial p_{j}^{i}}+\sum_{i=1}^{m} \sum_{j_{1}, j_{2}=1}^{n} p_{j_{1}, j_{2}}^{i} \frac{\partial}{\partial p_{j_{1} j_{2}}^{i}}\right. \\
& \left.+\cdots+\sum_{i=1}^{m} \sum_{j_{1}, \cdots, j_{k}=1}^{n} p_{j_{1} \cdots j_{k}}^{i} \frac{\partial}{\partial p_{j_{1} \cdots j_{k}}^{i}}\right)+\sum_{i=1}^{m} b_{i} \frac{\partial}{\partial \boldsymbol{z}_{i}} .
\end{aligned}
$$

Proof. For a function $\varphi$ defined on a neighbourhood of $j_{x}^{k}(f) \epsilon$ $J^{k}\left(R^{n}, E\right)$, we can define the function $\partial_{j}^{\#} \varphi$ by

$$
\begin{aligned}
\partial_{j}^{\#} \varphi= & \frac{\partial \varphi}{\partial x_{j}}+\sum_{i=1}^{m} p_{j}^{i} \frac{\partial \varphi}{\partial z_{i}}+\sum_{i=1}^{m} \sum_{j_{1}=1}^{n} p_{j j_{1}}^{i} \frac{\partial \varphi}{\partial p_{j_{1}}^{i}} \\
& +\cdots+\sum_{i=1}^{m} \sum_{j_{1}, \ldots, j_{k}=1}^{n} p_{j j_{1} \cdots j_{k}}^{i} \frac{\partial \varphi}{\partial p_{j_{1} \cdots j_{k}}^{i} \ldots} .
\end{aligned}
$$

Then we have $\partial_{j}^{\sharp} X=X^{(1)} \partial_{j}^{\#}$ and $\partial_{j}^{\sharp} X^{(k)}=X^{(k+1)} \partial_{j}^{\sharp}(j=1, \cdots, n ; k \geq 1)$ for any vector field $X$ on $E$ ([2], Lemma 2.3). In particular from $\partial_{j}^{\#} X z_{i}=$ $X^{(k+1)} \partial_{j}^{\#} z_{i} \quad(i=1, \cdots, m ; j=1, \cdots, n)$ and $\partial_{j}^{\#} X^{(k)} p_{j_{1} \cdots j_{k}}^{i}=X^{(k+1)} \partial_{j}^{\#} p_{j_{1} \cdots j_{k}}^{i}$ $\left(i=1, \cdots, m ; j_{1}, \cdots, j_{k}=1, \cdots, n ; k \geq 1\right)$, for the vector field $X=\sum_{i=1}^{m}$ $\left(a z_{i}+b_{i}\right) \partial / \partial z_{i}$ we inductively obtain

$$
\begin{aligned}
X^{(k)}= & a\left(\sum_{i=1}^{m} z_{i} \frac{\partial}{\partial \boldsymbol{z}_{i}}+\sum_{i=1}^{m} \sum_{j=1}^{n} p_{j}^{i} \frac{\partial}{\partial p_{j}^{i}}+\cdots\right. \\
& \left.+\sum_{i=1}^{m} \sum_{j_{1}, \cdots, j_{k}=1}^{n} p_{j_{1} \cdots j_{k}}^{i} \frac{\partial}{\partial p_{j_{1} \cdots j_{k}}^{i}}\right)+\sum_{i=1}^{m} b_{i} \frac{\partial}{\partial \boldsymbol{z}_{i}} .
\end{aligned}
$$

This completes the proof.

Denote by $\mathscr{G}$ the Lie algebra of all affine vector fields $X$ on $E$ of the form $X=\sum_{i=1}^{m}\left(a z_{i}+b_{i}\right) \partial / \partial z_{i}$ where $a$ and $b_{i} \in R$. A function $y$ locally defined at $j_{x}^{k}(f) \in J^{k}\left(R^{n}, E\right)$ is called a differential invariant of $\mathscr{G}$ at $j_{x}^{k}(f)$ if $X^{(k)} y=0$ for any $X \in \mathscr{G}$.

LEMma 4.2. Let $y$ be a function defined on a neighbourhood of $j_{x}^{k}(f)$ $\in J^{k}\left(R^{n}, E\right)$. Then $y$ is a differential invariant of $\mathscr{G}$ at $j_{x}^{k}(f)$ for $k \geq 1$ if and only if

$$
\begin{aligned}
\sum_{i=1}^{m} \sum_{j=1}^{n} p_{j}^{i} \frac{\partial y}{\partial p_{j}^{i}} & +\sum_{i=1}^{m} \sum_{j_{1}, j_{2}=1}^{n} p_{j_{1} j_{2}}^{i} \frac{\partial y}{\partial p_{j_{1} j_{2}}^{i}}+\cdots \\
& +\sum_{i=1}^{m} \sum_{j_{1}, \ldots, j_{k}=1}^{n} p_{j_{1} \cdots j_{k}}^{i} \frac{\partial y}{\partial p_{j_{1} \cdots, j_{k}}^{i}}=0
\end{aligned}
$$

and $\partial y / \partial z_{i}=0(i=1, \cdots, m)$.

Proof. By Lemma 4.1, $X^{(k)} y=0$ for any $X \in \mathscr{G}$ if and only if 


$$
\begin{aligned}
& a\left(\sum_{i=1}^{m} \boldsymbol{z}_{i} \frac{\partial y}{\partial \boldsymbol{z}_{i}}+\sum_{i=1}^{m} \sum_{j=1}^{n} \boldsymbol{p}_{j}^{i} \frac{\partial y}{\partial \boldsymbol{p}_{j}^{i}}+\cdots+\sum_{i=1}^{m} \sum_{j_{1}, \cdots, j_{k}=1}^{n} p_{j_{1} \cdots j_{k}}^{i} \frac{\partial y}{\partial p_{j_{1} \cdots j_{k}}^{i}}\right) \\
& \quad+\sum_{i=1}^{m} \boldsymbol{b}_{i} \frac{\partial \boldsymbol{y}}{\partial \boldsymbol{z}_{i}}=\mathbf{0}
\end{aligned}
$$

for any $a$ and $b_{i} \in R$. This implies Lemma 4.2.

LEMMA 4.3. Let $f$ be a local map of $R^{n}$ to $E$ such that $p_{11}^{1}\left(j_{x_{0}}^{2}(f)\right) \neq 0$. Then the sheaf of vector fields $\mathscr{L}$ generated by $\mathscr{G}$ is a weak Lie algebra sheaf which is $R^{n}$-regular at $\left(x_{0}, f\right)$.

Proof. Choose a sufficiently small neighbourhood $\mathscr{U}^{k}$ of $p_{0}=j_{x_{0}}^{k}(f)$, set $\mathscr{G}^{(k)}=\left\{X^{(k)} ; X \in \mathscr{G}\right\}$ and denote by $\mathscr{G}_{p}^{(k)}, p \in \mathscr{U}^{k}$, the isotropy algebra of $\mathscr{G}^{(k)}$ at $p$. Then by Lemma $4.1 \mathscr{G}_{p}^{(k)}=0$ and $\operatorname{dim} \mathscr{G}^{(k)}=m+1$. Since $\mathscr{L}^{(k)}$ is generated by $\mathscr{G}^{(k)}$, we get $(\mathscr{L})_{p}^{(k), 0}=0$ and $\operatorname{dim}(\mathscr{L})_{p}^{(k)}=\operatorname{dim} \mathscr{G}^{(k)}$ on a neighbourhood of $p_{0}$. Therefore $\mathscr{L}$ is $R^{n}$-regular at $\left(x_{0}, f\right)$. This completes the proof.

If $\mathscr{L}$ is $R^{n}$-regular at $\left(x_{0}, f\right)$, then it induces an involutive distribution $D^{(k)}$ on a neighbourhood of $j_{x_{0}}^{k}(f) \in J^{k}\left(R^{n}, E\right)$. A fundamental system of 1-st integrals of $D^{(k)}$ on a neighbourhood of $j_{x_{0}}^{k}(f)$ is called a fundamental system of differential invariants of $\mathscr{L}$ at $j_{x_{0}}^{k}(f)$.

LemMa 4.4. Let $\mathscr{L}$ be as in Lemma 4.3 and let $f$ be a local map of $R^{n}$ to $E$ such that $p_{11}^{1}\left(j_{x_{0}}^{2}(f)\right) \neq 0$. Then a family of functions

$$
\left\{x_{1}, \cdots, x_{n}, \cdots, p_{j}^{i} / p_{11}^{1}, \cdots, p_{j_{1} j_{2}}^{i} / p_{11}^{1}, \cdots\right\}
$$

locally defined at $j_{x_{0}}^{2}(f)$ is a fundamental system of differential invariants of $\mathscr{L}$ at $j_{x_{0}}^{2}(f)$.

Proof. From Lemma 4.2 we can easily see that $p_{j}^{i} / p_{11}^{1}(i=1, \cdots, m$; $j=1, \cdots, n)$ and $p_{j_{1} j_{2}}^{i} / p_{11}^{1}\left(i=1, \cdots, m ; j_{1}, j_{2}=1, \cdots, n ; j_{1}\right.$ or $\left.j_{2} \neq 1\right)$ are differential invariants of $\mathscr{L}$ at $j_{x_{0}}^{2}(f)$. Furthermore they are linearly independent at $j_{x_{0}}^{2}(f)$. They are $s$ functions where $s=m n+m n(n+1) / 2$ - 1. On the other hand $\operatorname{dim} J^{2}\left(R^{n}, E\right)=n+m+m n+m n(n+1) / 2$ and, for $p=j_{x_{0}}^{2}(f), \operatorname{dim}(\mathscr{L})_{p}^{(2)} /(\mathscr{L})_{p}^{(2), 0}=m+1$. Therefore $\operatorname{dim} J^{2}\left(R^{n}, E\right)-\operatorname{dim}(\mathscr{L})_{p}^{(2)} /$ $(\mathscr{L})_{p}^{(2), 0}=n+m n+m n(n+1) / 2-1$. This completes the proof.

For any differential operator $P(x, D)=\sum_{|p| \leq k} a_{p}(x) D^{p}$, we denote by $\mathscr{L}(P)$ the sheaf of vector fields on $E$ induced from the automorphism pseudogroup $\mathscr{A}(P)$ of $P(x, D)$ and denote by $P$ the differential equation $P(x, D) z=0$. If the differential equation 


$$
F_{1}=0, \cdots, F_{r}=0
$$

given by a family of functions $\left\{F_{j}\right\}_{j=1}^{r}$ locally defined at $j_{x_{0}}^{k}(f) \in J^{k}\left(R^{n}, E\right)$ admits the same space of local solutions as the differential equation $P$, the family $\left\{F_{j}\right\}_{j=1}^{r}$ is called a generator of $P(x, D)$ at $j_{x_{0}}^{k}(f)$.

Theorem 4.5. Let $f$ be a map of a neighbourhood of $x_{0}$ to $E$ such that $p_{11}^{i}\left(j_{x_{0}}^{2}(f)\right) \neq 0(i=1, \cdots, m)$. Assume that $P(x, D)=\sum_{p \leq 2} a_{p}(x) D^{p}$ is a projective elliptic differential operator. Then

(1) $\mathscr{L}(P)$ is a weak Lie algebra sheaf which is $R^{n}$-regular at $\left(x_{0}, f\right)$.

(2) $P(x, D)$ is generated at $j_{x_{0}}^{2}(f)$ by a family of functions $\left\{\mathscr{H}_{j}\right\}_{j=1}^{m}$ such that $\mathscr{H}_{j}=F_{j}\left(x_{1}, \cdots, x_{n}, \theta_{1}, \cdots, \theta_{N}\right)$ where $\left\{x_{1}, \cdots, x_{n}, \theta_{1}, \cdots, \theta_{N}\right\}$ is a fundamental system of differential invariants of $\mathscr{L}(P)$ at $j_{x_{0}}^{2}(f)$.

Proof. Since $P(x, D)$ is projective, $\mathscr{L}(P)$ is the sheaf of vector fields $\mathscr{L}$ generated by $\mathscr{G}$. Therefore by Lemma 4.3, (1) has been proved.

On a neighbourhood of $j_{x_{0}}^{2}(f)$, we set $\theta_{i, j}=p_{j}^{i} / p_{11}^{1}$ and $\theta_{i, j k}=p_{j k}^{i} / p_{11}^{1}$. By the ellipticity of $P(x, D)$, the differential equation $P$ is written by

$$
p_{11}^{i}=\sum_{j=1}^{m} \sum_{\substack{k, \ell=1 \\ k \neq 1 \\ o r \\ \ell \neq 1}}^{n} M_{k \ell}^{i j}(x) p_{k \ell}^{j}+\sum_{j=1}^{m} \sum_{\ell=1}^{n} N_{\ell}^{i j}(x) p_{\ell}^{j} \quad(i=1, \cdots, m) .
$$

Then on a neighbourhood of $j_{x_{0}}^{2}(f),(4.1)$ is written by

$$
\begin{gathered}
\sum_{\substack { j=1 \\
\begin{subarray}{c}{k, \ell=1 \\
k \neq 1 \\
\ell \neq 1{ j = 1 \\
\begin{subarray} { c } { k , \ell = 1 \\
k \neq 1 \\
\ell \neq 1 } }\end{subarray}}^{n} M_{k \ell}^{i j}(x)\left(p_{k \ell}^{j} / p_{11}^{i}\right)+\sum_{j=1}^{m} \sum_{\ell=1}^{n} N_{\ell}^{i j}(x)\left(p_{\ell}^{j} / p_{11}^{i}\right)-1=0 \\
(i=1, \cdots, m) .
\end{gathered}
$$

Since $p_{k \ell}^{j} / p_{11}^{i}=\left(p_{k \ell}^{j} / p_{11}^{1}\right) /\left(p_{11}^{i} / p_{11}^{1}\right)$ and $p_{\ell}^{j} / p_{11}^{i}=\left(p_{\ell}^{j} / p_{11}^{1}\right) /\left(p_{11}^{i} / p_{11}^{1}\right)$, (4.2) is written by

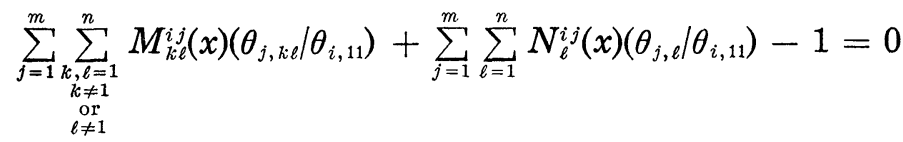

$$
\begin{aligned}
& (i=1, \cdots, m) .
\end{aligned}
$$

Then also by the projectivity of $P(x, D)$ and by Lemma 4.4, (2) has been proved. This completes the proof.

\section{§ 3. Equivalence problems of projective elliptic differential operators}

5. Let $G$ be the Lie subgroup of the affine transformation group on 
$E$ generated by $\mathscr{G}=\left\{\sum_{i=1}^{m}\left(a z_{i}+b_{i}\right) \partial / \partial z_{i} ; a\right.$ and $\left.b_{i} \in R\right\}$. Let $\Gamma$ denote the pseudogroup on $E$ generated by $G$ and let $\mathscr{N}(\Gamma)$ be the normalizer of $\Gamma$ in the pseudogroup of all local transformations of $E$. Denote by $\operatorname{Aff}(E)$ the affine transformation group on $E$.

Lemma 5.1. $\mathcal{N}(\Gamma)$ is generated by $\operatorname{Aff}(E)$.

Proof. Let $X$ be a local infinitesimal transformation such that the local 1-parameter group of local transformations generated by $X$ is contained in $\Gamma$. Then $X$ is written by

$$
X=\sum_{i=1}^{m}\left(\alpha z_{i}+\beta_{i}\right) \frac{\partial}{\partial z_{i}}
$$

where $\alpha$ and $\beta_{i}$ are some constants. For any $\phi \in \mathscr{N}(\Gamma)$, we set $\phi_{i}=z_{i} \circ \phi$. Then $\phi_{*} X$ is written by

$$
\phi_{*} X=\sum_{i=1}^{m}\left(\gamma \phi_{\imath}+\varepsilon_{i}\right) \frac{\partial}{\partial \phi_{\imath}}
$$

where $\gamma$ and $\varepsilon_{i}$ are some constants. If we use the Jacobi matrix of $\phi$ with respect to the coordinate system $\left\{z_{1}, \cdots, z_{m}\right\},(5.1)$ is written by

$$
\frac{D\left(\phi_{1}, \cdots, \phi_{m}\right)}{D\left(z_{1}, \cdots, z_{m}\right)}\left(\begin{array}{c}
\alpha z_{1}+\beta_{1} \\
\vdots \\
\alpha z_{m}+\beta_{m}
\end{array}\right)=\left(\begin{array}{c}
\gamma \phi_{1}+\varepsilon_{1} \\
\vdots \\
\gamma \phi_{m}+\hat{z}_{m}
\end{array}\right) \text {. }
$$

Therefore we get the relation

$$
\gamma \phi_{i}+\varepsilon_{i}=\alpha\left(\sum_{\ell=1}^{m} \frac{\partial \phi_{i}}{\partial \boldsymbol{z}_{\ell}} \boldsymbol{z}_{\ell}\right)+\sum_{p=1}^{m} \beta_{p} \frac{\partial \phi_{i}}{\partial \boldsymbol{z}_{p}} \quad(i=1, \cdots, m) .
$$

Differentiating (5.3), we obtain

$$
\begin{gathered}
\gamma \frac{\partial \phi_{i}}{\partial \boldsymbol{z}_{k}}=\alpha\left\{\sum_{\ell=1}^{m}\left(\frac{\partial^{2} \phi_{i}}{\partial \boldsymbol{z}_{k} \partial \boldsymbol{z}_{\ell}} \boldsymbol{z}_{\ell}+\frac{\partial \phi_{i}}{\partial \boldsymbol{z}_{\ell}} \delta_{k \ell}\right)\right\}+\sum_{p=1}^{m} \beta_{p} \frac{\partial^{2} \phi_{i}}{\partial \boldsymbol{z}_{k} \partial \boldsymbol{z}_{p}} \\
(i, k=1, \cdots, m) .
\end{gathered}
$$

Now assume that $\left(\partial \phi_{i} / \partial z_{k}\right) \neq 0$. Then rewriting (5.4) by

$$
\gamma=\left(\frac{\partial \phi_{i}}{\partial \boldsymbol{z}_{k}}\right)^{-1}\left\{\sum_{\ell=1}^{m}\left(\frac{\partial^{2} \phi_{i}}{\partial \boldsymbol{z}_{k} \partial \boldsymbol{z}_{\ell}} \boldsymbol{z}_{\ell}+\frac{\partial \phi_{i}}{\partial \boldsymbol{z}_{\ell}} \boldsymbol{\delta}_{k \ell}\right)\right\}+\sum_{p=1}^{m} \beta_{p}\left(\frac{\partial \phi_{i}}{\partial \boldsymbol{z}_{k}}\right)^{-1} \frac{\partial^{2} \phi_{i}}{\partial \boldsymbol{z}_{k} \partial \boldsymbol{z}_{p}}
$$

and differentiating (5.5), we get 


$$
\begin{gathered}
-\frac{\partial^{2} \phi_{i}}{\partial \boldsymbol{z}_{h} \partial \boldsymbol{z}_{k}}\left(\sum_{\ell=1}^{m}\left(\frac{\partial^{2} \boldsymbol{\phi}_{i}}{\partial \boldsymbol{z}_{k} \partial \boldsymbol{z}_{\ell}} \boldsymbol{z}_{\ell}+\frac{\partial \phi_{i}}{\partial \boldsymbol{z}_{\ell}} \hat{\boldsymbol{\delta}}_{k \ell}\right)\right) \\
+\frac{\partial \phi_{i}}{\partial \boldsymbol{z}_{k}}\left(\sum_{\ell=1}^{m}\left(\frac{\partial^{3} \boldsymbol{\phi}_{i}}{\partial \boldsymbol{z}_{h} \partial \boldsymbol{z}_{k} \partial \boldsymbol{z}_{\ell}} \boldsymbol{z}_{\ell}+\frac{\partial^{2} \boldsymbol{\phi}_{i}}{\partial \boldsymbol{z}_{k} \partial \boldsymbol{z}_{\ell}} \delta_{h \ell}+\frac{\partial^{2} \phi_{i}}{\partial \boldsymbol{z}_{h} \partial \boldsymbol{z}_{\ell}} \boldsymbol{\delta}_{k \ell}\right)\right) \\
+\sum_{p=1}^{m} \beta_{p}\left(-\frac{\partial^{2} \boldsymbol{\phi}_{i}}{\partial \boldsymbol{z}_{h} \partial \boldsymbol{z}_{k}} \frac{\partial^{2} \boldsymbol{\phi}_{i}}{\partial \boldsymbol{z}_{k} \partial \boldsymbol{z}_{p}}+\frac{\partial \phi_{i}}{\partial \boldsymbol{z}_{k}} \frac{\partial^{3} \boldsymbol{\phi}_{i}}{\partial \boldsymbol{z}_{h} \partial \boldsymbol{z}_{k} \partial \boldsymbol{z}_{p}}\right)=0 \\
(i, h, k=1, \cdots, m) .
\end{gathered}
$$

Since $\alpha$ and $\beta_{p}$ are arbitrary, we obtain

$$
\frac{\partial \phi_{i}}{\partial \boldsymbol{z}_{k}} \frac{\partial^{3} \phi_{i}}{\partial \boldsymbol{z}_{h} \partial \boldsymbol{z}_{k} \partial \boldsymbol{z}_{p}}=\frac{\partial^{2} \phi_{i}}{\partial \boldsymbol{z}_{h} \partial \boldsymbol{z}_{k}} \frac{\partial^{2} \phi_{i}}{\partial \boldsymbol{z}_{k} \partial \boldsymbol{z}_{p}}
$$

and

$$
\begin{gathered}
\sum_{\ell=1}^{m} \frac{\partial \phi_{i}}{\partial \boldsymbol{z}_{k}}\left(\frac{\partial^{3} \boldsymbol{\phi}_{i}}{\partial \boldsymbol{z}_{h} \partial \boldsymbol{z}_{k} \partial \boldsymbol{z}_{\ell}} \boldsymbol{z}_{\ell}+\frac{\partial^{2} \boldsymbol{\phi}_{i}}{\partial \boldsymbol{z}_{k} \partial \boldsymbol{z}_{\ell}} \boldsymbol{\delta}_{h \ell}+\frac{\partial^{2} \boldsymbol{\phi}_{i}}{\partial \boldsymbol{z}_{h} \partial \boldsymbol{z}_{\ell}} \boldsymbol{\delta}_{k \ell}\right) \\
=\sum_{\ell=1}^{m}-\frac{\partial^{2} \boldsymbol{\phi}_{i}}{\partial \boldsymbol{z}_{h} \partial \boldsymbol{z}_{k}}\left(\frac{\partial^{2} \phi_{i}}{\partial \boldsymbol{z}_{k} \partial \boldsymbol{z}_{\ell}} \boldsymbol{z}_{\ell}+\frac{\partial \phi_{i}}{\partial \boldsymbol{z}_{\ell}} \boldsymbol{\delta}_{k \ell}\right) \\
(i, h, k=1, \cdots, m)
\end{gathered}
$$

which are reduced to the relations

$$
\begin{gathered}
\sum_{\ell=1}^{m} \frac{\partial \phi_{i}}{\partial \boldsymbol{z}_{k}}\left(\frac{\partial^{2} \phi_{i}}{\partial \boldsymbol{z}_{k} \partial \boldsymbol{z}_{\ell}} \boldsymbol{\delta}_{h \ell}+\frac{\partial^{2} \phi_{i}}{\partial \boldsymbol{z}_{h} \partial \boldsymbol{z}_{\ell}} \boldsymbol{\delta}_{k \ell}\right)=\sum_{\ell=1}^{m} \frac{\partial^{2} \phi_{i}}{\partial \boldsymbol{z}_{h} \partial \boldsymbol{z}_{k}} \frac{\partial \phi_{i}}{\partial \boldsymbol{z}_{\ell}} \\
(i, h, k=1, \cdots, m) .
\end{gathered}
$$

Then we get

$$
\frac{\partial \phi_{i}}{\partial z_{k}} \frac{\partial^{2} \phi_{i}}{\partial z_{k} \partial z_{h}}=0 \quad(i, h, k=1, \cdots, m) \quad \text { i.e. }
$$

$\partial \phi_{i} / \partial \boldsymbol{z}_{k}$ is constant for any $i$ and $k$. Therefore in any case $\partial \phi_{i} / \partial \boldsymbol{z}_{k}$ is constant and so $\phi$ is an affine transformation. Conversely it is clear that Aff $(E)$ is contained in $\mathscr{N}(\Gamma)$. This completes the proof of Lemma 5.1.

Let $\alpha^{2}$ (resp. $\beta^{2}$ ) denote the source projection (resp. the target projection) of $J^{2}\left(R^{n}, E\right)$ onto $R^{n}$ (resp. $E$ ). If we set $J_{x, z}^{2}=\left\{p \in J^{2}\left(R^{n}, E\right)\right.$; $\left.\alpha^{2}(p)=x, \beta^{2}(p)=z\right\}$, then $J_{x, z}^{2}$ admits the globally defined coordinate system $\left\{\cdots, p_{j}^{i}, \cdots, p_{k j}^{i}, \cdots\right\}$ and is diffeomorphic to $R^{\bar{N}}$ where $\bar{N}=$ $m(n+n(n+1) / 2)$. Therefore we can consider that the family of functions $\left\{\cdots, p_{j}^{i}, \cdots, p_{j k}^{i}, \cdots\right\}$ is the homogeneous coordinate system of the projective space $P^{N}(R)$ where $N=\bar{N}-1$. 
TheOREM 5.2. Assume that $p_{11}^{i}\left(j_{x_{0}}^{2}(f)\right) \neq 0(i=1, \cdots, m)$. Let $P_{\alpha}(x, D)$ $(\alpha=1,2)$ be projective elliptic differential operators of order 2 . Then $P_{1}(x, D)$ is locally isomorphic to $P_{2}(x, D)$ at $\left(f\left(x_{0}\right), f\left(x_{0}\right)\right)$ by a local isomorphism near to the identity if and only if there exists a generator $\left\{F_{(\alpha) r}\left(x_{1}, \cdots, x_{n}\right.\right.$, $\left.\left.\theta_{1}, \cdots, \theta_{N}\right)\right\}_{r=1}^{m}$ of $P_{\alpha}(x, D)$ at $j_{x_{0}}^{2}(f)$ satisfying the following property: $B y$ considering $F_{(\alpha) r}^{x}$, defined by $F_{(\alpha) r}^{x}\left(\theta_{1}, \cdots, \theta_{N}\right)=F_{(\alpha) r}\left(x_{1}, \cdots, x_{n}, \theta_{1}, \cdots, \theta_{N}\right)$, as a local function on the projective space $P^{N}(R)$, there is a local projective transformation $\varphi$ near to the identity of $P^{N}(R)$ such that

$$
\begin{aligned}
& \varphi^{*} F_{(2) r}^{x}=F_{(1) r}^{x} \quad(r=1, \cdots, m), \\
& \varphi^{*} \theta_{i, j}=\left(\sum_{k=1}^{m} q_{i k} \theta_{k, j}\right) /\left(\sum_{k=1}^{m} q_{1 k} \theta_{k, 11}\right)
\end{aligned}
$$

and

$$
\varphi^{*} \theta_{i, j \ell}=\left(\sum_{k=1}^{m} q_{i k} \theta_{k, j \ell}\right) /\left(\sum_{k=1}^{m} q_{1 k} \theta_{k, 11}\right)
$$

where $\left(q_{i j}\right)$ is an $m \times m$ regular matrix.

Proof. Let $\phi: \mathscr{U} \rightarrow \mathscr{V}$ be a local isomorphism of $P_{1}(x, D)$ to $P_{2}(x, D)$ at $\left(f\left(x_{0}\right), f\left(x_{0}\right)\right)$. Then $\phi\left(\mathscr{S}\left(P_{1}\right) \mid \mathscr{U}\right)=\mathscr{S}\left(P_{2}\right) \mid \mathscr{U}$ and so we have $\phi^{-1}\left(\mathscr{A}\left(P_{1}\right) \mid \mathscr{V}\right) \phi$ $=\mathscr{A}\left(P_{2}\right) \mid \mathscr{U}$. Since $P_{\alpha}(x, D) \quad(\alpha=1,2)$ is projective, this means that $\phi \in \mathscr{N}(\Gamma)$. Then by Lemma 5.1 we get

$$
\begin{aligned}
& \phi^{*} z_{i}=\sum_{k=1}^{m} q_{i k} z_{k}+d_{i}, \\
& \phi^{(1) *} p_{j}^{i}=\sum_{k=1}^{m} q_{i k} p_{j}^{k}
\end{aligned}
$$

and

$$
\phi^{(2) *} p_{j \ell}^{i}=\sum_{k=1}^{m} q_{i k} p_{j \ell}^{k} .
$$

Since $\phi^{(2)}$ maps a sufficiently small neighbourhood $\mathscr{U}^{2}$ of $j_{x_{0}}^{2}(f)$ to an open subset of $J^{2}\left(R^{n}, E\right)$ such that $\phi^{(2) *} p_{11}^{i}(p) \neq 0$ for any $p \in \mathscr{U}^{2}$, we get

$$
\begin{aligned}
\phi^{(1) * \theta_{i, j}} & =\left(\phi^{(1) *} p_{j}^{i}\right) /\left(\phi^{(2) *} p_{11}^{1}\right) \\
& =\left(\sum_{k=1}^{m} q_{i k} p_{j}^{k}\right) /\left(\sum_{k=1}^{m} q_{1 k} p_{11}^{k}\right) \\
& =\left(\sum_{k=1}^{m} q_{i k} \theta_{k, j}\right) /\left(\sum_{k=1}^{m} q_{1 k} \theta_{k, 11}\right)
\end{aligned}
$$

and 


$$
\begin{aligned}
\phi^{(2) *} \theta_{i, j \ell} & =\left(\phi^{(2) *} p_{j \ell}^{i}\right) /\left(\phi^{(2) *} p_{11}^{1}\right) \\
& =\left(\sum_{k=1}^{m} q_{\imath k} \theta_{k, j \ell}\right) /\left(\sum_{k=1}^{m} q_{1 k} \theta_{k, 11}\right) .
\end{aligned}
$$

If $\left\{F_{(2) r}\left(x_{1}, \cdots, x_{n}, \theta_{1}, \cdots, \theta_{N}\right)\right\}_{r=1}^{m}$ is a generator of $P_{2}(x, D)$, then, by setting $F_{(1) r}\left(x_{1}, \cdots, x_{n}, \theta_{1}, \cdots, \theta_{N}\right)=\phi^{(2) *} F_{(2) r}\left(x_{1}, \cdots, x_{n}, \theta_{1}, \cdots, \theta_{N}\right),\left\{F_{(1) r}\left(x_{1}, \cdots, x_{n}\right.\right.$, $\left.\left.\theta_{1}, \cdots, \theta_{N}\right)\right\}_{r=1}^{m}$ is a generator of $P_{1}(x, D)$. (5.10) and (5.11) mean that $\phi$ induces a local projective transformation $\varphi$ of $P^{N}(R)$ because $\left\{\cdots, \theta_{i, j}, \cdots\right.$, $\left.\theta_{i, j k}, \cdots\right\}$ can be considered as a local coordinate system of the projective space $P^{N}(R)$.

Conversely if there exists a local projective transformation $\varphi$ of $P^{N}(R)$ satisfying the stated property, then the affine transformation $\phi=\left(\phi_{1}, \cdots, \phi_{m}\right)$ on $E$ defined by $\phi_{i}=\sum_{k=1}^{m} q_{i k} z_{k}+d_{i}$ where $d_{i}(i=1, \cdots, m)$ is any constant satisfies $\phi^{(2) *} F_{(2) r}=F_{(1) r}$ and $\phi$ is a local isomorphism of $P_{1}(x, D)$ to $P_{2}(x, D)$ at $\left(f\left(x_{0}\right), f\left(x_{0}\right)\right)$. This completes the proof of Theorem 5.2.

Let us consider regular submanifolds $M$ of $P^{N}(R)$ satisfying the following conditions:

(1) $\operatorname{codim} M=m$.

(2) At any point $p \in M$, there exists a system of local defining equations of $M$

$$
\eta_{1}=0, \cdots, \eta_{m}=0
$$

such that the automorphism pseudogroup $\mathscr{A}$ of the differential equation

$$
\eta_{1}\left(\theta_{1}, \cdots, \theta_{N}\right)=0, \cdots, \eta_{m}\left(\theta_{1}, \cdots, \theta_{N}\right)=0
$$

is equal to $\Gamma$.

Denote by $\mathscr{C}(\Gamma)$ the set of such regular submanifolds of $P^{N}(R)$.

Remark 5.1. Theorem 5.2 implies that the local equivalence of pseudoinvolutive projective elliptic differential operators of order 2 can be reduced to that of elements in $\mathscr{C}(\Gamma)$ smoothly parametrized by $x=$ $\left(x_{1}, \cdots, x_{n}\right)$ under the group

$$
H=\left\{\left(\begin{array}{ccc}
g & & 0 \\
& g & \\
0 & \cdot & \cdot g
\end{array}\right\}: g \in G L(m, R)\right\} / \begin{gathered}
\text { The center } \\
\text { of } \\
G L(N+1, R) .
\end{gathered}
$$

6. For a projective elliptic differential operator $P(x, D)$ of order 2 (4.1) is called the normal form of $P$ and (4.2) is called the canonical form 
of $P$. The left hand side of (4.2) is called the canonical generator of $P(x, D)$.

Let $P_{\alpha}(x, D)(\alpha=1,2)$ be a pseudoinvolutive projective elliptic differential operator. Denote the canonical form of $P_{\alpha}$ by

$$
\begin{gathered}
\sum_{j=1}^{m} \sum_{\substack{k, \ell=1 \\
\text { or } \\
\ell \neq 1}}^{n} M_{\alpha k \ell}^{\lambda j}(x)\left(p_{k \ell}^{j} / p_{11}^{\lambda}\right)+\sum_{j=1}^{m} \sum_{k=1}^{n} N_{\alpha k}^{\lambda j}(x)\left(p_{k}^{j} / p_{11}^{\lambda}\right)-1=0 \\
(\lambda=1, \cdots, m) .
\end{gathered}
$$

LEMMA 6.1. $\mathscr{S}\left(P_{1}\right)=\mathscr{S}\left(P_{2}\right)$ if and only if $M_{1 k \ell}^{\lambda j}(x) \equiv M_{2 k \ell}^{\lambda j}(x) \quad(\lambda, j=$ $1, \cdots, m ; k, \ell=1, \cdots, n ; k$ or $\ell \neq 1)$ and $N_{1 k}^{\lambda j}(x) \equiv N_{2 k}^{\lambda j}(x)(\lambda, j=1, \cdots, m$; $k=1, \cdots, n)$ for each $x$.

Proof. The relation (6.1) holds on $S\left(P_{\alpha}\right)$ and if $P_{1}(x, D)=P_{2}(x, D)$, then $S\left(P_{1}\right)=S\left(P_{2}\right)=I$ and codim $I=m$. From $(6.1)_{1}$ and $(6.1)_{2}$, we get

$$
\begin{gathered}
\sum_{j=1}^{m} \sum_{\substack{k, \ell=1 \\
\text { or } \\
\ell \neq 1}}^{n}\left(M_{1 k \ell}^{\lambda j}(x)-M_{2 k \ell}^{\lambda j}(x)\right) p_{k \ell}^{j}+\sum_{j=1}^{m} \sum_{k=1}^{n}\left(N_{1 k}^{\lambda j}(x)-N_{2 k}^{\lambda j}(x)\right) p_{k}^{j}=0 \\
\text { on } I(\lambda=1, \cdots, m) .
\end{gathered}
$$

Since $p_{k \ell}^{j}(j=1, \cdots, m ; k, \ell=1, \cdots, n ; k$ or $\ell \neq 1)$ and $p_{k}^{j}(j=1, \cdots, m$; $k=1, \cdots, n)$ are independent on $I$, from (6.2) we have $M_{1 k \ell}^{\lambda j}(x) \equiv M_{2 k \ell}^{\lambda j}(x)$ $(\lambda, j=1, \cdots, m ; k, \ell=1, \cdots, n ; k$ or $\ell \neq 1)$ and $N_{1 k}^{\lambda j}(x) \equiv N_{2 k}^{\lambda j}(x) \quad(\lambda, j=$ $1, \cdots, m ; k=1, \cdots, n)$. The converse assertion obviously holds. This has proved Lemma 6.1.

Corollary 6.2. Let $P_{\alpha}(x, D)(\alpha=1,2)$ be a pseudoinvolutive projective elliptic differential operator of order 2 and assume that $p_{11}^{i}\left(j_{x_{0}}^{2}(f)\right) \neq 0$ $(i=1, \cdots, m)$. Then $P_{1}(x, D)$ is locally isomorphic to $P_{2}(x, D)$ at $\left(f\left(x_{0}\right), f\left(x_{0}\right)\right)$ by a local isomorphism near to the identity if and only if there exists a regular $m \times m$ matrix $Q=\left(q_{i j}\right)$ near to the unit such that $Q^{-1} M_{k \ell}^{(2)} Q=M_{k \ell}^{(1)}$ $(k, \ell=1, \cdots, n ; k$ or $\ell \neq 1)$ and $Q^{-1} N_{k}^{(2)} Q=N_{k}^{(1)}(k=1, \cdots, n)$ where $M_{k \ell}^{(\alpha)}$ (resp. $N_{k}^{(\alpha)}$ ) is a function defined on a neighbourhood of $x_{0}$ such that the value $M_{k l}^{(\alpha)}(x)$ (resp. $N_{k}^{(\alpha)}(x)$ ) at $x$ is the $m \times m$ matrix whose $(i, j)$ component is $M_{\alpha k \varepsilon}^{i j}(x)$ (resp. $N_{\alpha k}^{i j}(x)$ ).

Proof. By Lemma $5.1 P_{1}(x, D)$ is locally isomorphic to $P_{2}(x, D)$ at $\left(f\left(x_{0}\right), f\left(x_{0}\right)\right)$ by a local isomorphism near to the identity if and only if there exists an affine transformation $\phi=\left(\phi_{1}, \cdots, \phi_{m}\right), \phi_{i}(z)=\sum_{k=1}^{m} q_{i k} z_{k}+r_{i}$, 
such that $\phi$ is near to the identity and $\phi\left(\mathscr{P}\left(P_{1}\right) \mid \mathscr{U}\right)=\mathscr{S}\left(P_{2}\right) \mid \mathscr{V}$ where $\mathscr{U}$ and $\mathscr{V}$ are neighbourhoods of $f\left(x_{0}\right)$. Then for any $u \in \mathscr{S}\left(P_{1}\right) \mid \mathscr{U}$, we have

$$
-A_{11}^{(1)}(x) \frac{\partial^{2} u}{\partial x_{1}^{2}}=\sum_{\substack{k, \ell=1 \\ k \neq 1 \\ \text { or } \\ \ell \neq 1}}^{n} A_{k \ell}^{(1)}(x) \frac{\partial^{2} u}{\partial x_{k} \partial x_{\ell}}+\sum_{k=1}^{n} B_{k}^{(1)}(x) \frac{\partial u}{\partial x_{k}}
$$

and

$$
-A_{11}^{(2)}(x)\left(Q \frac{\partial^{2} u}{\partial x_{1}^{2}}\right)=\sum_{\substack{k, \ell=1 \\ k \neq 1 \\ \text { or } \\ \ell \neq 1}}^{n} A_{k \ell}^{(2)}(x)\left(Q \frac{\partial^{2} u}{\partial x_{k} \partial x_{\ell}}\right)+\sum_{k=1}^{n} B_{k}^{(2)}(x)\left(Q \frac{\partial u}{\partial x_{k}}\right)
$$

where $\sum_{k, \ell=1}^{n} A_{k \ell}^{(\alpha)}(x)\left(\partial^{2} u / \partial x_{k} \partial x_{\ell}\right)+\sum_{k=1}^{n} B_{k}^{(\alpha)}(x)\left(\partial u / \partial x_{k}\right)=0$ is the coordinate expression of the differential equation $P$ and $Q=\left(q_{i j}\right)$. The solution space of (6.3) is the same as that of (6.4) and therefore by considering the canonical forms of (6.3) and (6.4), Lemma 6.1 shows that $Q^{-1} M_{k \ell}^{(2)} \boldsymbol{Q}=$ $M_{k \ell}^{(1)}(k, \ell=1, \cdots, n ; k$ or $\ell \neq 1)$ and $Q^{-1} N_{k}^{(2)} Q=N_{k}^{(1)}(k=1, \cdots, n)$. This completes the proof.

\section{§4. Existence of projective elliptic differential operators}

7. Define the subset $G \subset G L(n, R)$ by $G \ni X=\left(x_{i j}\right)$ if and only if $\sum_{k=1}^{n} x_{k \delta}=\sum_{k=1}^{n} x_{k 1} \quad(\delta \geq 1)$ and consider the map $c: G \ni X=\left(x_{i j}\right) \rightarrow$ $\sum_{k=1}^{n} x_{k 1} \in R$ and set $R_{*}=R-\{0\}$.

LEMMA 7.1. $G$ is a subgroup of $G L(n, R)$ and $c$ is a homomorphism of $G$ onto $R_{*}$.

Proof. If $G \ni X=\left(x_{i j}\right)$ and $G L(n, R) \ni Y=\left(y_{i j}\right)$ such that $X Y=I$ where $I$ is the unit matrix $\in G L(n, R)$, we have $\sum_{i=1}^{n} \sum_{k=1}^{n} x_{i k} y_{k j}=\sum_{i=1}^{n} \delta_{i j}=1$. On the other hand $\sum_{i=1}^{n} \sum_{k=1}^{n} x_{i k} y_{k j}=\sum_{k=1}^{n}\left(\sum_{i=1}^{n} x_{i k}\right) y_{k j}=\sum_{k=1}^{n}\left(\sum_{i=1}^{n} x_{i 1}\right) y_{k j}=$ $\left(\sum_{i=1}^{n} x_{i 1}\right)\left(\sum_{k=1}^{n} y_{k j}\right)$. Thus $\left(\sum_{i=1}^{n} x_{i 1}\right)\left(\sum_{k=1}^{n} y_{k j}\right)=1$. This means that $\sum_{k=1}^{n} y_{k j}$ is independent of $j$ i.e. $\sum_{k=1}^{n} y_{k \jmath}=\sum_{k=1}^{n} y_{k 1}$ which proves $Y=X^{-1} \in G$. If $G \ni X=\left(x_{i j}\right)$ and $Y=\left(y_{i j}\right)$, then $\sum_{k=1}^{n} x_{k j}=\sum_{k=1}^{n} x_{k 1}$ and $\sum_{k=1}^{n} y_{k j}=\sum_{k=1}^{n} y_{k 1}$ $(j=1, \cdots, n)$. Then by setting $X Y=\left(z_{i j}\right), \sum_{k=1}^{n} z_{k j}=\sum_{k=1}^{n}\left(\sum_{h=1}^{n} x_{k h} y_{h j}\right)=$ $\sum_{h=1}^{n}\left(\sum_{k=1}^{n} x_{k h}\right) y_{h J}=\sum_{h=1}^{n}\left(\sum_{k=1}^{n} x_{k 1}\right) y_{h j}=\left(\sum_{k=1}^{n} x_{k 1}\right)\left(\sum_{h=1}^{n} y_{h 1}\right)=\sum_{h=1}^{n}\left(\sum_{k=1}^{n} x_{k 1}\right) y_{h 1}$ $=\sum_{h=1}^{n}\left(\sum_{k=1}^{n} x_{k h} y_{h 1}\right)=\sum_{k=1}^{n} z_{k 1}$. This proves $X Y \in G$. Therefore $G$ is a subgroup of $G L(n, R)$.

If $G \ni X=\left(x_{i j}\right)$, it is clear that $\sum_{k=1}^{n} x_{k 1} \neq 0$. Furthermore for $X=\left(x_{i j}\right)$ and $Y=\left(y_{i j}\right) \in G$, we have $\sum_{i=1}^{n}\left(\sum_{k=1}^{n} x_{i k} y_{k 1}\right)=\left(\sum_{i=1}^{n} x_{i 1}\right)\left(\sum_{k=1}^{n} y_{k 1}\right)$. Therefore $c$ is a homomorphism of $G$ onto $R_{*}$. The proof is completed. 
8. Let $P(x, D)=\sum_{|p| \leq 2} a_{p}(x) D^{p}$ be an elliptic differential operator of $\Gamma\left(R^{n}, E\right)$ to $\Gamma\left(R^{n}, F\right)$ where $\operatorname{dim} E=\operatorname{dim} F=m$.

For some $j, P(x, D)$ is said to satisfy the condition $C(j)$ if $m=n$ and $\operatorname{det}\left(t_{i k}(x)\right) \neq 0$ where $t_{i k}(x)=b_{k}^{i j}(x)$ and $b_{k}^{i j}(x)$ is the coefficient appearing in (3.1).

Proposition 8.1. Let $P(x, D)=\sum_{|p| \leq 2} a_{p}(x) D^{p}$ be an elliptic pseudoinvolutive differential operator such that $b_{k}^{i 1}(x) \equiv b_{k}^{i j}(x) \quad(i=1, \cdots, m$; $j=2, \cdots, m ; k=1, \cdots, n), c^{i j}(x) \equiv 0(i, j=1, \cdots, m)$ and the condition $C(1)$ is satisfied. Then $P(x, D)$ is projective.

Proof. Since $c^{i j}(x) \equiv 0(i, j=1, \cdots, m)$, by Proposition 3.1, (3.2) is reduced to

$$
\sum_{\alpha, j=1}^{m}\left(\sum_{k, \ell=1}^{n} a_{k}^{i j}(x) \frac{\partial^{2} u_{\alpha}}{\partial x_{k} \partial x_{\ell}}+\sum_{k=1}^{n} b_{k}^{i j}(x) \frac{\partial u_{\alpha}}{\partial x_{k}}\right) \frac{\partial \phi_{j}}{\partial u_{\alpha}}=0 \quad(i=1, \cdots, m)
$$

on $S(P)=I(P)$.

Furthermore since $P(x, D)$ satisfies the condition $C(1)$, solving (3.1) with respect to $\partial u_{1} / \partial x_{k}(k=1, \cdots, m)$, we get

$$
\frac{\partial u_{1}}{\partial x_{k}}=\sum_{j, \alpha, \beta=1}^{m} F_{\alpha \beta}^{k j}(x) \frac{\partial^{2} u_{j}}{\partial x_{\alpha} \partial x_{\beta}}+\sum_{j=2}^{m} \sum_{\ell=1}^{m} G_{\ell}^{k j}(x) \frac{\partial u_{j}}{\partial x_{\ell}} \quad(k=1, \cdots, m)
$$

on $S(P)=I(P)$.

Since $\partial^{2} u_{j} / \partial x_{\alpha} \partial x_{\beta} \quad(j, \alpha, \beta=1, \cdots, m)$ and $\partial u_{j} / \partial x_{r} \quad(j=2, \cdots, m ; \gamma=$ $1, \cdots, m)$ are independent on $I(P)$, from (8.1) and (8.2), we get as the system of defining equations of $\mathscr{A}(P)$,

$$
\begin{gathered}
\sum_{j=1}^{m}\left(a_{\alpha \beta}^{i j}(x)+\sum_{\gamma=1}^{m} b_{r}^{i j}(x) F_{\alpha \beta}^{r 1}(x)\right) \frac{\partial \phi_{j}}{\partial u_{1}}=0 \\
(\alpha, \beta, i=1, \cdots, m), \\
\sum_{j=1}^{m}\left(\sum_{\gamma=1}^{m} b_{\gamma}^{i j}(x) F_{\alpha \beta}^{\gamma \delta}(x) \frac{\partial \phi_{j}}{\partial u_{1}}+a_{\alpha \beta}^{i j}(x) \frac{\partial \phi_{j}}{\partial u_{\delta}}\right)=0 \\
(\alpha, \beta, i=1, \cdots, m ; \delta=2, \cdots, m), \\
\sum_{j=1}^{m}\left(\sum_{\gamma=1}^{m} b_{r}^{i j}(x) G_{\ell}^{r \delta}(x) \frac{\partial \phi_{j}}{\partial u_{1}}+b_{\ell}^{i j}(x) \frac{\partial \phi_{j}}{\partial u_{\delta}}\right)=0 \\
(\ell, i=1, \cdots, m ; \delta=2, \cdots, m) .
\end{gathered}
$$

We define $m \times m$ matrices by

$$
\begin{gathered}
H_{\alpha \beta}(x)=\left(H_{\alpha \beta}^{i j}(x)\right) \quad \text { where } H_{\alpha \beta}^{i j}(x)=a_{\alpha \beta}^{i j}(x)+\sum_{k=1}^{m} b_{k}^{i j}(x) F_{\alpha \beta}^{k 1}(x) \\
(\alpha, \beta=1, \cdots, m),
\end{gathered}
$$




$$
\begin{aligned}
& K_{\alpha \beta \delta}(x)=\left(K_{\alpha \beta \delta}^{i j}(x)\right) \quad \text { where } \quad K_{\alpha \beta \delta}^{i j}(x)=\sum_{k=1}^{m} b_{k}^{i j}(x) F_{\alpha \beta}^{k \delta}(x) \\
&(\alpha, \beta=1, \cdots, m ; \delta=2, \cdots, m), \\
& S_{\delta \ell}(x)=\left(S_{i \ell}^{i j}(x)\right) \quad \text { where } \quad S_{\dot{\delta} \ell}^{i j}(x)=\sum_{k=1}^{m} b_{k}^{i j}(x) G_{\ell}^{k \delta}(x) \\
&(\ell=1, \cdots, m ; \delta=2, \cdots, m), \\
& A_{\alpha \beta}(x)=\left(a_{\alpha \beta}^{i j}(x)\right) \quad(\alpha, \beta=1, \cdots, m)
\end{aligned}
$$

and

$$
B_{\ell}(x)=\left(b_{\ell}^{i j}(x)\right) \quad(\ell=1, \cdots, m) .
$$

Then (8.3) is written by

$$
\begin{array}{ll}
H_{\alpha \beta}(x) w_{1}=0 & (\alpha, \beta=1, \cdots, m), \\
K_{\alpha \beta \delta}(x) w_{1}+A_{\alpha \beta}(x) w_{\delta}=0 & (\alpha, \beta=1, \cdots, m ; \delta=2, \cdots, m), \\
S_{\delta \ell}(x) w_{1}+B_{\ell}(x) w_{\delta}=0 & (\ell=1, \cdots, m ; \delta=2, \cdots, m)
\end{array}
$$

where $w_{k}={ }^{t}\left(\partial \phi_{1} / \partial u_{k}, \cdots, \partial \phi_{m} / \partial u_{k}\right)$.

By the condition $b_{k}^{i j}(x)=b_{k}^{i 1}(x)(i, k=1, \cdots, m ; j=2, \cdots, m)$, an easy calculation shows that $H_{\alpha \beta}^{i j}(x)=a_{\alpha \beta}^{i j}(x)-a_{\alpha \beta}^{i 1}(x)(i, j, \alpha, \beta=1, \cdots, m), K_{\alpha \beta}^{i j}(x)$ $=-a_{\alpha \beta}^{i \delta}(x)(i, j, \alpha, \beta=1, \cdots, m ; \delta=2, \cdots, m)$ and $S_{\delta \ell}^{i j}(x)=-b_{\ell}^{i 1}(x)(i, j, \ell$ $=1, \cdots, m ; \delta=2, \cdots, m)$. Therefore (8.4) is reduced to

$$
\begin{aligned}
& \sum_{k=1}^{n}\left(a_{\alpha \beta}^{i k}(x)-a_{\alpha \beta}^{i 1}(x)\right) w_{k 1}=0 \quad(i, \alpha, \beta=1, \cdots, m), \\
& \sum_{k=1}^{m} a_{\alpha \beta}^{i k}(x) w_{k \delta}-a_{\alpha \beta}^{i \delta}(x)\left(\sum_{k=1}^{m} w_{k 1}\right)=0 \\
& (i, \alpha, \beta=1, \cdots, m ; \delta=2, \cdots, m), \\
& \sum_{k=1}^{m} w_{k \delta}-\sum_{k=1}^{m} w_{k 1}=0 \quad(\delta=2, \cdots, m)
\end{aligned}
$$

where

$$
w_{k}={ }^{t}\left(w_{1 k}, \cdots, w_{m k}\right)={ }^{t}\left(\frac{\partial \phi_{1}}{\partial u_{k}}, \cdots, \frac{\partial \phi_{m}}{\partial u_{k}}\right)
$$

From the second and the third relations of (8.5), we get

$$
\sum_{k=1}^{m}\left(a_{\alpha \beta}^{i k}(x)-a_{\alpha \beta}^{i \delta}(x)\right) w_{k \delta}=0 \quad(i, \alpha, \beta=1, \cdots, m ; \delta=2, \cdots, m) .
$$

Therefore the system of defining equations (8.5) of $\mathscr{A}(P)$ is equivalent to 


$$
\begin{aligned}
& \sum_{k=1}^{m}\left(a_{\alpha \beta}^{i k}(x)-a_{\alpha \beta}^{i \delta}(x)\right) w_{k \delta}=0 \quad(i, \alpha, \beta, \delta=1, \cdots, m), \\
& \sum_{k=1}^{m} w_{k \delta}=\sum_{k=1}^{m} w_{k 1} \quad(\delta=1, \cdots, m) .
\end{aligned}
$$

Since $\phi=\left(\phi_{1}, \cdots, \phi_{m}\right)$ is an affine transformation of $E$, the second relation of (8.6) means that the matrix $\left(\partial \phi_{i} / \partial u_{j}\right)$ belongs to the group $G$ given in Lemma 7.1. Define the map $\pi_{\delta}: G L(m, R) \rightarrow R^{m}$ by the $\delta$-th column projection. Then from (8.6) we get

$$
A_{\alpha \beta}(x) \pi_{\delta}(w)=c(w) \pi_{\dot{\delta}}\left(A_{\alpha \beta}(x)\right) \quad(\delta=1, \cdots, m)
$$

where $w=\left(w_{1}, \cdots, w_{m}\right)$ and $c$ is the homomorphism of $G$ onto $R_{*}$ given in Lemma 7.1.

From (8.7) if $c(w)=1$, then we have $A_{\alpha \beta}(x) \pi_{\delta}(w)=\pi_{\delta}\left(A_{\alpha \beta}(x)\right) \quad(\delta=$ $1, \cdots, m$ ) which mean that $w=I$. Therefore $c: L \mathscr{A}_{0}(P) \rightarrow R_{*}$ is an isomorphism and this proves that $L \mathscr{A}_{0}(P)$ is the center of $G L(m, R)$. Conversely if $L \mathscr{A}_{0}(P)$ is the center of $G L(m, R)$, then each element $\phi$ of $\mathscr{A}(P)$ satisfies (8.6). Since $c^{i j}(x) \equiv 0(i, j=1, \cdots, m)$, this completes the proof of Proposition 8.1.

Proposition 8.2. For an elliptic pseudoinvolutive differential operator $P(x, D)=\sum_{|p| \leq 2} a_{p}(x) D^{p}$ satisfying the condition $C(1)$, assume that $b_{k}^{i j}(x) \equiv 0$ $(i, k=1, \cdots, m ; j=2, \cdots, m)$ and $c^{i j}(x) \equiv 0(i, j=1, \cdots, m)$. Then $P(x, D)$ is projective.

Proof. From the conditions $b_{k}^{i j}(x)=0(i, k=1, \cdots, m ; j=2, \cdots, m)$, we can easily see that $H_{\alpha \beta}^{i 1}(x)=0 \quad(i, \alpha, \beta=1, \cdots, m), H_{\alpha \beta}^{i j}(x)=a_{\alpha \beta}^{i j}(x)$ $(i, \alpha, \beta=1, \cdots, m ; j=2, \cdots, m), \quad K_{\alpha \beta \delta}^{i j}(x)=0 \quad(i, \alpha, \beta=1, \cdots, m ; \delta, j=$ $2, \cdots, m), K_{\alpha \beta \delta}^{i 1}(x)=-a_{\alpha \beta}^{i \delta}(x)(i, \alpha, \beta=1, \cdots, m ; \delta=2, \cdots, m)$ and $S_{\delta \dot{\delta}}^{i j}(x)=0$ $(i, j, \ell=1, \cdots, m ; \delta=2, \cdots, m)$. Then (8.4) is reduced to

$$
\begin{array}{ll}
\sum_{j=2}^{m} a_{\alpha \beta}^{i j}(x) w_{j 1}=0 & (i, \alpha, \beta=1, \cdots, m), \\
\sum_{j=1}^{m} a_{\alpha \beta}^{i j}(x) w_{j \delta}=a_{\alpha \beta}^{i \delta}(x) w_{11} & (i, \alpha, \beta=1, \cdots, m ; \delta=2, \cdots, m), \\
b_{\ell}^{i 1} w_{1 \delta}=0 & (i, \ell=1, \cdots, m ; \delta=2, \cdots, m) .
\end{array}
$$

The third relation of (8.8) and the condition $C(1)$ mean that $w_{1 \delta}=0$ $(\delta=2, \cdots, m)$. Therefore (8.8) is equivalent to 


$$
\begin{array}{ll}
w_{1 \delta}=0 & (\delta=2, \cdots, m) \\
A_{\alpha \beta}(x) w_{\delta}=w_{11} \pi_{\delta}\left(A_{\alpha \beta}(x)\right) & (\alpha, \beta, \delta=1, \cdots, m)
\end{array}
$$

where

$$
w_{k}={ }^{t}\left(w_{1 k}, \cdots, w_{m k}\right) \text {. }
$$

Denote by $G^{\prime}$ the subgroup of $G L(m, R)$ defined by $G^{\prime} \ni X=\left(x_{i j}\right)$ if and only if $x_{1 j}=0(j=2, \cdots, m)$ and denote by $c^{\prime}$ the homomorphism of $G$ to $R_{*}$ defined by $c^{\prime}(X)=x_{11}$. Then (8.9) is written by

$$
\begin{aligned}
& w_{1 \delta}=0 \quad(\delta=2, \cdots, m), \\
& A_{\alpha \beta}(x) w_{\delta}=c^{\prime}(w) \pi_{\delta}\left(A_{\alpha \beta}(x)\right) \quad(\alpha, \beta, \delta=1, \cdots, m)
\end{aligned}
$$

where

$$
w=\left(w_{1}, \cdots, w_{m}\right)
$$

If $c^{\prime}(w)=1$, then (8.10) means that $w=I$ i.e. $c^{\prime}$ is an isomorphism of $L \mathscr{A}_{0}(P)$ onto $R_{*}$. Therefore $L \mathscr{A}_{0}(P)$ is the center of $G L(E)$. This completes the proof.

9. Finally we shall refer, in the case $m<n$, to the existence of a projective elliptic differential operator of order 2 .

An elliptic differential operator $P(x, D)$ of order 2 is said to satisfy the condition $C^{\prime}(j)$ if $m<n$ and $\operatorname{det} B^{j}(x) \neq 0$ where $B^{j}(x)$ is the $m \times m$ matrix whose $(i, k)$-component is $b_{k}^{i j}(x)$

Proposition 9.1. For an elliptic pseudoinvolutive differential operator $P(x, D)$ of order 2 satisfying the conditions $C^{\prime}(1)$ and $c^{i j}(x) \equiv 0(i, j=$ $1, \cdots, m)$, assume that $b_{k}^{i 1}(x) \equiv b_{k}^{i j}(x) \quad(i=1, \cdots, m ; j=2, \cdots, m ; k=$ $1, \cdots, n)$ or $b_{k}^{i j}(x) \equiv 0(i=1, \cdots, m ; j=2, \cdots, m ; k=1, \cdots, n)$. Then $P(x, D)$ is projective if and only if $b_{h}^{i 1}(x) \equiv 0 \quad(i=1, \cdots, m ; h=m+$ $1, \cdots, n)$.

Proof. By the condition $C^{\prime}(1),(8.2)$ is replaced by

$$
\begin{aligned}
\frac{\partial u_{1}}{\partial x_{k}}= & \sum_{j=1}^{m} \sum_{\alpha, \beta=1}^{n} F_{\alpha \beta}^{k j}(x) \frac{\partial^{2} u_{j}}{\partial x_{\alpha} \partial x_{\beta}}+\sum_{j=2}^{m} \sum_{\ell=1}^{n} G_{\ell}^{k j}(x) \frac{\partial u_{j}}{\partial x_{\ell}} \\
& +\sum_{h=m+1}^{n} H_{h}^{k}(x) \frac{\partial u_{1}}{\partial x_{h}} \quad(k=1, \cdots, m)
\end{aligned}
$$

on $S(P)=I(P)$. Then from (8.1) and (9.1) as the system of defining equations of $\mathscr{A}(P)$, we get the following systems of differential equations (9.2) and (9.3): 


$$
\begin{aligned}
& \sum_{j=1}^{m}\left(a_{\alpha \beta}^{i j}(x)+\sum_{\gamma=1}^{m} b_{r}^{i j}(x) F_{\alpha \beta}^{r 1}(x)\right) \frac{\partial \phi_{j}}{\partial u_{1}}=0 \\
& (i=1, \cdots, m ; \alpha, \beta=1, \cdots, n), \\
& \sum_{j=1}^{m}\left(\sum_{r=1}^{m} b_{r}^{i j}(x) F_{\alpha \beta}^{r \delta}(x) \frac{\partial \phi_{j}}{\partial u_{1}}+\boldsymbol{a}_{\alpha \beta}^{i j}(x) \frac{\partial \phi_{j}}{\partial u_{\delta}}\right)=0 \\
& (i=1, \cdots, m ; \alpha, \beta=1, \cdots, n ; \delta=2, \cdots, m), \\
& \sum_{j=1}^{m}\left(\sum_{\gamma=1}^{m} b_{\gamma}^{i j}(x) G_{\ell}^{r \delta}(x) \frac{\partial \phi_{j}}{\partial u_{1}}+b_{\ell}^{i j}(x) \frac{\partial \phi_{j}}{\partial u_{\delta}}\right)=0 \\
& (i=1, \cdots, m ; \ell=1, \cdots, n ; \delta=2, \cdots, m)
\end{aligned}
$$

and

$$
\begin{gathered}
\sum_{k, j=1}^{m} b_{k}^{i j}(x) H_{h}^{k}(x) \frac{\partial \phi_{j}}{\partial u_{1}}=0 \\
(i=1, \cdots, m ; h=m+1, \cdots, n) .
\end{gathered}
$$

If we set $H_{h}^{i j}(x)=\sum_{k=1}^{m} b_{k}^{i j}(x) H_{h}^{k}(x)$ and denote by $H_{h}(x)$ the $m \times m$ matrix whose $(i, j)$-component is $H_{h}^{i j}(x)$, then (9.3) is written by

$$
H_{h}(x) w_{1}=0 \quad(h=m+1, \cdots, n)
$$

where

$$
w_{j}={ }^{t}\left(\frac{\partial \phi_{1}}{\partial u_{j}}, \cdots, \frac{\partial \phi_{m}}{\partial u_{j}}\right)
$$

Now by the condition $C^{\prime}(1)$ we can set $b_{h}^{i 1}(x)=\sum_{k=1}^{m} c_{h}^{k}(x) b_{k}^{i 1}(x)$ for $i=1, \cdots, m$ and $h=m+1, \cdots, n$. Then it is easy to see that

$$
H_{h}^{k}(x) \equiv-c_{h}^{k}(x) \quad(k=1, \cdots, m ; h=m+1, \cdots, n) .
$$

Since $w=\left(w_{1}, \cdots, w_{m}\right)$ satisfies (9.2), as is proved in Proposition 8.1 or $8.2, w$ belongs to the center of $G L(m, R)$. In particular, $w_{1}$ is of the form ${ }^{t}(*, 0, \cdots, 0)$. Therefore any such $w_{1}$ satisfies (9.4) if and only if the 1-st column of $H_{h}(x)$ is zero i.e. $H_{h}^{i 1}(x) \equiv 0$. Since $H_{h}^{i 1}(x)=\sum_{k=1}^{m} b_{k}^{i 1}(x) H_{h}^{k}(x)$ and the condition $C^{\prime}(1)$ is satisfied, $H_{h}^{i 1}(x) \equiv 0 \quad(i=1, \cdots, m ; h=m+$ $1, \cdots, n)$ if and only if $H_{h}^{k}(x) \equiv 0(k=1, \cdots, m ; h=m+1, \cdots, n)$. Then by (9.5), $w_{1}$ satisfies (9.4) if and only if $c_{h}^{k}(x) \equiv 0(k=1, \cdots, m ; h=m+$ $1, \cdots, n)$ i.e. $b_{h}^{i 1}(x) \equiv 0(i=1, \cdots, m ; h=m+1, \cdots, n)$. This prove that the solution space of the system of differential equations (9.2) is equal to that of the system (9.2) with (9.3) if and only if $b_{h}^{i 1}(x) \equiv 0(i=1, \cdots, m$; $h=m+1, \cdots, n)$. This completes the proof of Proposition 9.1. 


\section{REFERENCES}

[1] I. M. Singer and S. Sternberg, The infinite groups of Lie and Cartan, J. Analyse Math., 15 (1965), 1-114.

[2] K. Ueno, Existence and equivalence theorems of automorphic systems, Publ. RIMS, Kyoto Univ., 11 (1976), 461-482.

Tokyo University of Fisheries Minato-ku, Tokyo 108, Japan 